\title{
Balloon rupture during stent postdilation: Lesson from a movie
}

\author{
Andreas Y Andreou MD
}

AY Andreou. Balloon rupture during stent postdilation: Lesson from a movie. Curr Res Cardiol 2016;3(1):25-26.

Balloon rupture (BR) is an infrequent complication of percutaneous coronary intervention (PCI) and can lead to vessel injury. The present article describes a case in which PCI for a lesion that did not appear to be severely calcified on angiography was complicated by BR during stent postdilation. Balloon rupture went unrecognized, with resultant coronary dissection.

\section{CASE PRESENTATION}

A 70-year-old diabetic man underwent coronary angiography due to atypical angina and exercise-induced ST-segment depression in leads V4 through V6. Angiography revealed a large, obtuse marginal branch $(\mathrm{OMB})$ that was severely obstructed by a discrete, mildly calcified lesion (Figure 1), which was addressed with percutaneous coronary intervention $(\mathrm{PCI})$. The lesion was predilated with a $2.0 \mathrm{~mm}$ $\times 9.0 \mathrm{~mm}$ semicompliant balloon inflated to 16 atmospheres (atm). Subsequently, a $3.0 \mathrm{~mm} \times 15 \mathrm{~mm}$ bare-metal stent was successfully implanted across the lesion at $10 \mathrm{~atm}$. However, during stent postdilation using a $3.0 \mathrm{~mm} \times 10 \mathrm{~mm}$ noncompliant balloon rated at $22 \mathrm{~atm}$, a sudden loss of pressure occurred at $12 \mathrm{~atm}$, with simultaneous leakage of contrast medium (CM) into a small stent-jailed sub-branch,

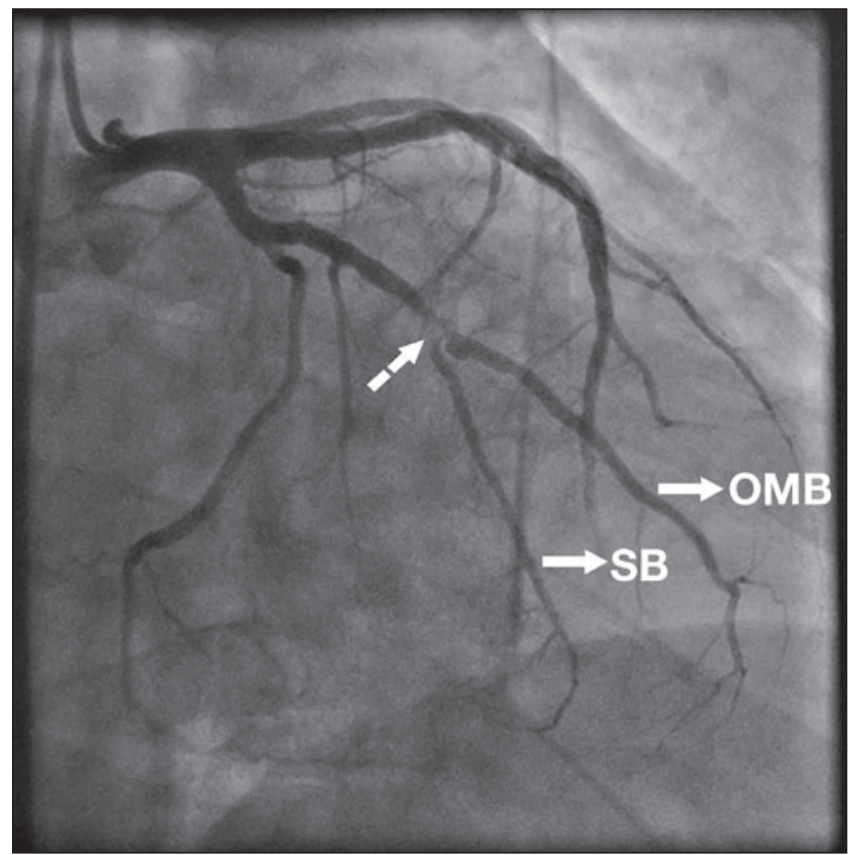

Figure 1) Coronary angiographic image from the right anterior oblique caudal projection revealing a significantly obstructive calcified lesion (dashed arrow) in the obtuse marginal branch (OMB) and a small side branch (SB) arising within the lesion
The presence of sharp calcium spicules - which could not be detected by angiography - is highlighted as the likely cause of BR. Vigilance for BR during every step of PCI, particularly in calcified lesions, is also emphasized along with the need for prompt recognition of BR to avoid or minimize coronary wall trauma.

Key Words: Balloon rupture; Coronary angiography; Coronary angioplasty; Coronary dissection; Percutaneous coronary intervention

suggesting balloon rupture (BR). The latter was not immediately realized and the balloon was further inflated to $18 \mathrm{~atm}$, resulting in further 2 leakage and accumulation of CM extraluminally at the site of balloon inflation and intraluminally immediately proximal to the balloon (Figure 2, Video 1). The balloon was then immediately deflated and retrieved without difficulty. Although control angiography revealed normal OMB blood flow, persisting extralum$\square$ inal extravasation of CM (confined within the stented segment) was compatible with type C coronary dissection (Figure 3, Video 2). No further intervention was undertaken and an eventual angiogram documented a good result with complete resolution of the dissection (Figure 4, Video 3). The patient tolerated the procedure well without chest pain or electrocardiographic

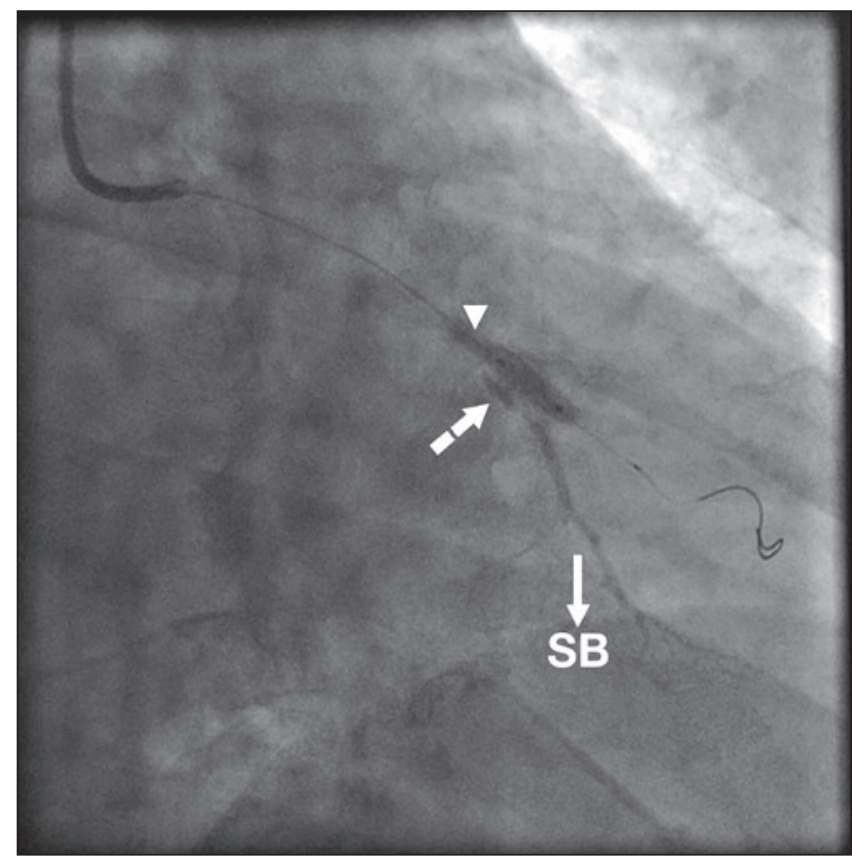

Figure 2) Fluoroscopic image depicting the ruptured balloon with leakage of contrast medium into the side branch (SB), extraluminally at the site of balloon inflation (dashed arrow) and intraluminally just proximal to the balloon (arrowhead)

Institutional Affiliation: Department of Cardiology, Limassol General Hospital, Limassol, Cyprus

Correspondence: Dr Andreas Y Andreou, Department of Cardiology, Limassol General Hospital, PO Box 56060, Nikeas Street,

Pano Polemidia 3304, Limassol, Cyprus. Telephone 357-25801437, fax 357-25801432, e-mail y.andreas@yahoo.com 


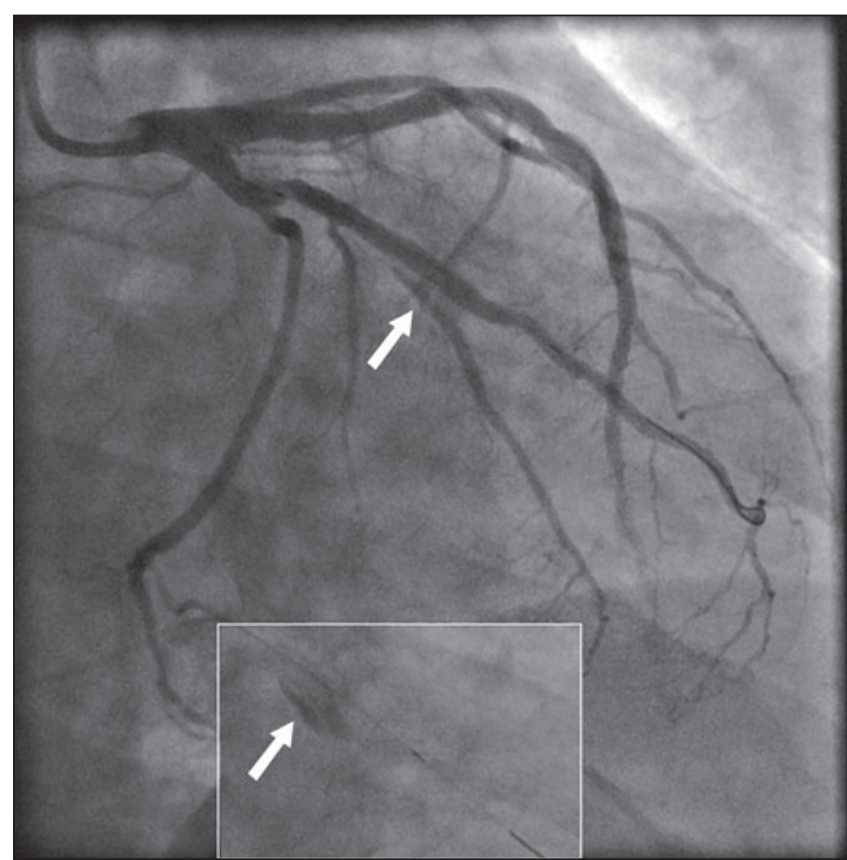

Figure 3) Coronary angiographic image from the right anterior oblique caudal projection revealing persisting extraluminal extravasation of contrast medium (embedded panel, arrow) being compatible with type C coronary dissection (arrow)

evidence of ischemia, and no evidence of post-PCI myocardial infarction. He was discharged home after an uneventful two-day hospital course, and remained asymptomatic and free of cardiac events two years poststenting.

\section{DISCUSSION}

$\mathrm{B}$ $\mathrm{R}$ is a rare, mostly benign complication of PCI, potentially leading to serious sequelae such as coronary perforation or dissection, air embolism or balloon entrapment (1-4). Among 1139 coronary lesions treated by PCI, Alfonso et al (1) described BR in 66 (5.8\%) lesions. In 50 patients, BR did not result in angiographically evident coronary wall trauma. However, a coronary dissection was detected in $16(24 \%)$ of these patients and, in two of these individuals, BR occurred during stent postdilation. Three patients died due to coronary perforation with cardiac tamponade, acute myocardial infarction and distal embolization of the balloon material, respectively. In a study by Simpfendorfer et al (2) involving 1500 PCIs, there were 55 (3.6\%) cases of BR. Coronary bypass graft surgery was performed in three cases due to a flow-limiting intimal dissection $(n=2)$ and acute vessel closure $(n=1)$.

BR has been associated with balloon oversizing/overinflation and lesion calcification, in which rupture usually occurs with normal pressures when sharp calcium spicules cut into the balloon surface (2-4). $\mathrm{BR}$ is recognized when a sudden loss in pressure occurs during balloon inflation or when the target pressure cannot be maintained (1). In the present case, BR occurred forcefully at a moderate pressure into the stent-jailed sub-branch, suggesting a pinhole perforation in the balloon material. Had we immediately recognized BR by the sudden loss in pressure of the indeflator - which coincided with opacification of the stent-jailed sub-branch by the leaking CM - and immediately deflated the balloon, the coronary dissection caused by further inflation of the already ruptured balloon at higher pressures would have

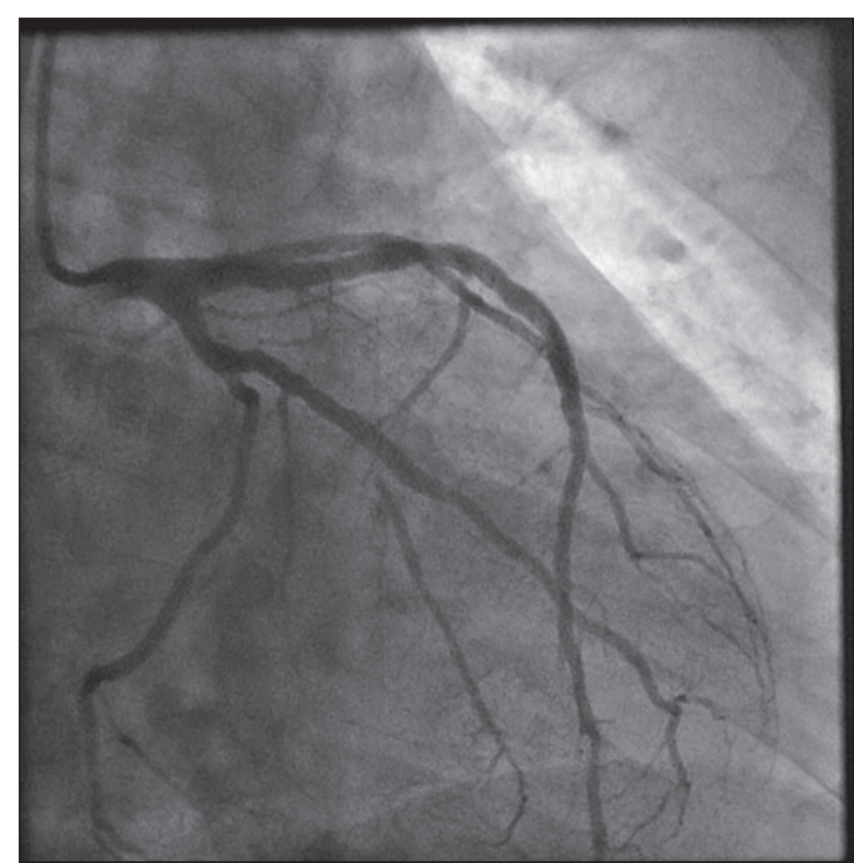

Figure 4) Coronary angiographic image in the right anterior oblique caudal projection revealing a good final result without evidence of dissection

been avoided or minimized. Furthermore, although lesion calcification was recognized on the angiogram before PCI, it was not severe, and the predilating balloon was fully inflated without waist or signs of rupture; therefore, subsequent BR during stent optimization was not anticipated. The lesion very likely contained jagged calcifications, which, upon stent implantation, became protruded through the stent struts and punctured the postdilating balloon. If the predilating balloon failed to inflate fully or rupture, we would have adopted a different strategy, such as intravascular ultrasound interrogation of the lesion followed by predilation with more specialized balloons, or debulking with rotational atherectomy. Nevertheless, repeat stent postdilation was not considered because angiography did not show frank stent underexpansion, and a possible repeat BR would risk extension of the dissection and/or coronary rupture. Conventional coronary angiography is inherently insufficient to appreciate complex features of atherosclerotic lesions, particularly calcification. Accordingly, vigilance for $\mathrm{BR}$ is required even if severe calcification is not detected on angiography because sharp calcium spicules penetrating into the lumen may be present. Unlike the present case, prompt recognition of $\mathrm{BR}$ followed by immediate deflation of the balloon may be all that is needed to prevent severe coronary trauma.

\section{REFERENCES}

1. Alfonso F, Pérez-Vizcayno MJ, Hernández R, et al. Clinical and angiographic implications of balloon rupture during coronary stenting. Am J Cardiol 1997;80:1077-80.

2. Simpfendorfer CC, Dimas AP, Zaidi A, et al. Balloon rupture during coronary angioplasty. Angiology 1986;37:828-31.

3. Zellner C, Sweeney JP, Ko E, Sudhir K, Chou TM. Use of intravascular ultrasound in evaluating repeated balloon rupture during coronary stenting. Cathet Cardiovasc Diagn 1997;40:52-4.

4. Macías Garrido E, Medina Vega MÁ, Gonzalo N, Escaned J.

High definition OCT image of sharp calcium spicule.

EuroIntervention 2014;10:601 